Décadrages Décadrages

cinéma, à travers champs Cinéma, à travers champs

$18 \mid 2011$

Mario Ruspoli et le « cinéma direct »

\title{
Pepperminta : hypnose colorée entre vidéo et cinéma
}

\section{Melissa Rérat}

\section{(2) OpenEdition}

1 Journals

Édition électronique

URL : https://journals.openedition.org/decadrages/232

DOI : $10.4000 /$ decadrages. 232

ISSN : 2297-5977

Éditeur

Association Décadrages

Édition imprimée

Date de publication : 10 avril 2011

Pagination : 127-136

ISBN : 978-2-9700668-2-8

ISSN : 2235-7823

Référence électronique

Melissa Rérat, « Pepperminta : hypnose colorée entre vidéo et cinéma », Décadrages [En ligne], 18|

2011, mis en ligne le 10 avril 2012, consulté le 03 avril 2022. URL : http://journals.openedition.org/ decadrages/232; DOI : https://doi.org/10.4000/decadrages.232 


\section{Pepperminta: hypnose colorée entre vidéo}

\section{et cinéma}

par Melissa Rérat ${ }^{1}$

Pepperminta (2009) est le premier long métrage de la vidéaste suisse Pipilotti Rist (fig. 1). Présenté en salles de cinéma et en festivals, il est toutefois difficile de le considérer uniquement et entièrement comme cinématographique. Le malaise ou l'hésitation s'expliquent principalement par la carrière artistique de la réalisatrice et par le contenu, la structure et l'esthétique du film. Habituée aux expositions en galeries et en musées, Pipilotti Rist franchit la ligne de démarcation, certes de plus en plus fine et floue à l'heure actuelle, entre le monde de l'art contemporain et celui du cinéma. Comme elle le déclare dans le dossier de presse accompagnant la sortie $\mathrm{du}$ film ${ }^{2}$, l'une de ses principales ambitions en s'essayant au cinéma était de toucher un public autre que celui de ses vidéos. Mais ce n'est pas pour autant qu'elle abandonne le langage visuel et les thématiques qui lui sont chères. Pepperminta regorge de couleurs, d'images et de symboles rappelant plusieurs de ses œuvres vidéo et installations. Pepperminta ne constitue pas tant un véritable saut dans le domaine du cinéma qu'une poursuite, une adaptation d'un univers artistique d'un médium à un autre, d'un dispositif à un autre. Pour ce faire, l'artiste a dû ne pas avoir peur de l'erreur. Ce sont le décalage, la faute, le flottement entre plusieurs médiums qui l'intéressent. Elle suit en cela le conseil prodigué à Pepperminta par sa grandmère: "Fais toujours ce que tu n'oses pas et regarde ce qu'il se passe.»3

Le propos de la présente étude est tout d'abord de faire le point sur l'accueil mitigé du film et de retracer les raisons qui ont conduit à l'incompréhension d'une partie du public. Il s'agira de montrer comment un horizon d'attente trop rigide met à mal l'entre-deux dans lequel se place le film. Enfin, la présentation de la logique intertextuelle et intermédiale $\mathbf{4}$ à l'œuvre tant dans le long métrage que dans d'autres travaux de Pipilotti Rist permettra d'établir la spécificité de Pepperminta.

\section{Pipilotti Rist au cinéma: un accueil mitigé}

Un parcours à travers les pages consacrées à Pepperminta tant dans la
1 Je tiens à remercier les personnes suivantes pour l'aide précieuse qu'elles m'ont apportée durant l'écriture de cet article et dans la phase de recherche qui l'a précédée: Karin Seinsoth de la galerie Hauser \& Wirth Zurich, Christof Neracher de Hugofilm Productions, Tamara Voser de l'Atelier Rist Sisters et François Bovier pour son travail de relecture.

2 «Pendant les 15 dernières années durant lesquelles j'ai réalisé des vidéos et des films expérimentaux pour mes installations audiovisuelles, j'ai développé une envie grandissante de réaliser un long métrage pour le cinéma. Pepperminta est aussi conçu pour un public extérieur au monde de l'art." (Je traduis à partir du dossier de presse, p. 18, réalisé par The Match Factory, diffuseur mondial du film et responsable des relations de presse internationales, disponible à l'adresse internet suivante: www.the-match-factory.com/films/items/ pepperminta.html).

3 Propos qui apparaît à 3' 17" après le début du film (je traduis). Toutes les références au film renvoient au dvd diffusé par The Match Factory: Pipilotti Rist, Pepperminta, Zurich/Vienne, Hugofilm Productions/Coop99, 2009, DVD PAL, 80 ', allemand avec sous-titres anglais.

4 Pour la question complexe de l'intermédialité, voir André Gaudreault, François Jost (éd.), La Croisée des médias, Paris, CREDHESS, 2000, et en particulier à l'article de Rick Altman "Technologie et textualité de l'intermédialité", pp. 11, 13: "[...] le terme intermédialité> se rapporterait à la fusion des médias, de même qu'à la confusion des spectateurs. [...] l'identité d'un média est à tel point mise en question que ce média en vient à s'apparenter à plusieurs des médias existants. C'est cette multiplicité d'identités qui rend possible l'intermédialité au sens fort et propre du terme.". 
5 La presse romande a réservé un accueil plus négatif que sa voisine alémanique qui se reflète dans le nombre d'entrées qu'a fait le film: 14437 pour la Suisse allemande, 485 pour la région romande (voir le site internet ProCinema, Association Suisse des exploitants et distributeurs de films: www.procinema.ch/f/ index.html).

6 Elisabeth Chardon, "Pipilotti Rist, du musée au ciné ", Le Temps, 3 février 2010, p. 26; voir également Gerhard Mack, "Im Paradies der Pop-Ära ", Neue Zürcher Zeitung, n ${ }^{\circ}$ 5, 30 août 2009, p. 47.

7 Stéphane Gobbo, "Le fantasme du cinéma", La Liberté, 5 février 2010, p. 34; Antoine Duplan, "Fifi Brindacier au pays de Sgt. Pepper's", L'Hebdo, 3 février 2010.

8 Le "mode de représentation institutionnel" tel que l'a défini Noël Burch dans La Lucarne de l'infini renvoie à ce que je qualifie de "cinéma commercial conventionnel». Burch situe l'établissement de ce mode filmique dominant dans les années 1910: "[...] un Mode de Représentation Institutionnel [...] que tous, tant que nous sommes, nous intériorisons très jeunes en tant que compétence de lecture grâce à une expérience des films (en salle ou à la télévision) à présent universellement précoce dans les sociétés industrielles. " (Noël Burch, La Lucarne de l'infini, Paris, Nathan, 1991, pp. 6-7).

9 «L'industrie du cinéma est prête pour un film qui questionne les esthétiques et la narration conventionnelles et qui, comme Pepperminta, innove. [...] Nous sommes convaincus que Pepperminta ne va pas susciter que l'intérêt des amateurs d'art mais a le potentiel de mener à une grande communauté de fans." (Je traduis à partir du dossier de presse, op. cit., p. 10).

10 Pepperminta a été présenté dans plusieurs festivals. Il a été sélectionné de manière récur- presse quotidienne que spécialisée, alémanique et romande 5 , permet de constater deux positions bien tranchées. Les journalistes issus du monde de l'art ou de la sphère culturelle célèbrent le film comme un passage réussi du musée au cinéma, attesté par l'inscription du style, de la "patte spécifique» 6 de Pipilotti Rist. A l'inverse, les critiques de cinéma n'apprécient guère cette intrusion vidéographique dans leur domaine ${ }^{7}$. Tous les commentateurs s'accordent cependant sur un point. Ils reconnaissent dans la forme, l'esthétique de Pepperminta une transposition de l'univers vidéo de Pipilotti Rist. Les admirateurs des vidéos et des installations de l'artiste saluent cette reprise. Leurs critères artistiques semblent leur faire oublier qu'il s'agit d'un long métrage cinématographique et non pas d'une vidéo artistique augmentée. Les critiques de cinéma s'empressent quant à eux de rappeler l'artiste à l'ordre. L'empreinte vidéo n'est nullement considérée comme positive mais dénoncée comme un raccourci facile emprunté par la réalisatrice qui n’a pas réussi à répondre aux exigences du cinéma. L'opposition binaire concernant la forme se retrouve dans l'analyse du fond. Les critiques positives considèrent l'histoire comme la clé permettant à la vidéo de pénétrer dans le domaine du cinéma. Certains journalistes vont même jusqu'à considérer la narrativité comme l'unique élément, avec la durée de quatre-vingts minutes, qui différencie Pepperminta d'une vidéo ou d'une installation audiovisuelle. La simple présence de l'histoire permet d'assimiler Pepperminta à un long métrage de cinéma innovateur. Un tel raccourci est inenvisageable pour les critiques de cinéma. Ils reconnaissent également dans la narration une innovation par rapport aux œuvres antérieures de l'artiste, mais la seule tentative ne suffit pas. Une brève analyse de l'histoire révèle selon eux son inconsistance, son manque de profondeur, la superficialité de thématiques soi-disant anticonformistes et une morale peu convaincante et des plus redondantes.

La lecture de ces articles montre que tous les critiques se basent sur les mêmes éléments, la poursuite d'un langage visuel provenant de la vidéo et la présence d'une histoire, mais ils en tirent des conclusions différentes. L'écart s'explique par les grilles de lecture employées pour appréhender Pepperminta. Les uns ont recours à des critères artistiques et recherchent la signature de l'artiste, alors que les autres emploient les outils d'analyse d'un cinéma qui reconduit un mode de représentation institutionnalisé 8 . Les deux points de vue manquent d'impartialité et d'objectivité. Ni totalement artistique ni totalement cinématographique, Pepperminta nécessite de recourir à des outils de lecture adaptés à son hybridité. 


\section{Grilles de lecture}

Le dossier de presse accompagnant la sortie du film insiste à plusieurs reprises sur les liens qu'entretient le long métrage avec la vidéo et l'installation. Les déclarations de Pipilotti Rist proposent également plusieurs rapprochements entre cinéma et vidéo. Quant au producteur du film, la firme zurichoise Hugofilm, il présente Pepperminta comme «un film qui questionne les esthétiques et la narration conventionnelles» 9 . Ce film a été présenté comme une création proche de l'art contemporain qui bouleverse les conventions cinématographiques dans le cadre de plusieurs festivals $\mathbf{1 0}$.

Avec son premier long métrage, Pipilotti Rist s'inscrit dans un mouvement d'appropriation et d'exploration du cinéma par l'art contemporain qui se développe dès les années 199011. Cette zone de rencontre entre Beaux-Arts et septième art est vaste et difficilement définissable avec précision. Le premier à s'être intéressé aux modalités de dialogue entre différents domaines de création, à cet espace de l'«entre», a été Raymond Bellour 12. Plus récemment, Pascale Cassagnau a proposé le concept de "troisième cinéma" pour qualifier l'ensemble des interactions entre cinéma et arts plastiques, littérature et danse contemporaine:

"[...] un territoire artistique, celui d'un autre cinéma, entre art contemporain, nouveaux médias, cinéma et documentaire: un troisième cinéma, qui ne saurait se confondre avec le cinéma expérimental ni avec l'art vidéo. $\mathbf{1 3}$

C'est bien avec ce territoire d'un cinéma de l'entre-deux, de la rencontre entre domaines de création que joue Pipilotti Rist14. Dans son travail

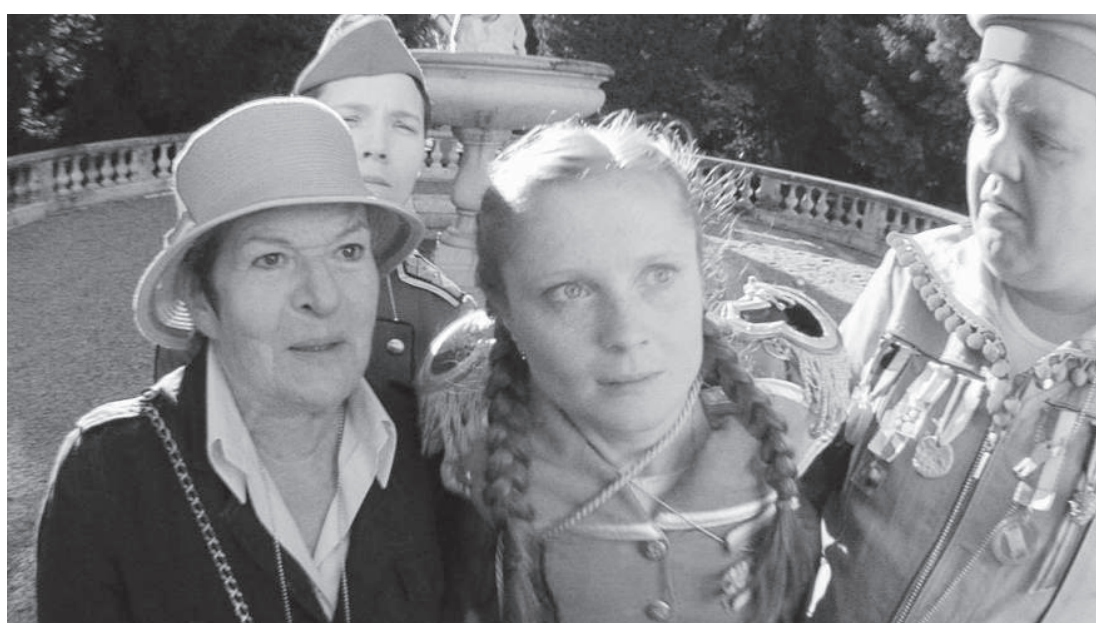

1/ Source: Frenetic Films rente dans des catégories réservées à des créations originales, bouleversant les distinctions entre médiums et proposant des expériences nouvelles aux spectateurs: La catégorie "Cutting The Edge" du $27^{\mathrm{e}}$ Festival International du Film de Miami, "New Frontier" au Sundance Film Festival 2010 de Park City, la compétition parallèle "Horizons" du 66 $6^{\mathrm{e}}$ Festival International du Film de Venise, la section "Bright Future" du 39e Festival International du Film de Rotterdam. II a remporté le prix extraordinaire du président du jury au Festival du Film Européen de Séville de 2009.

11 On peut songer aux installations de Douglas Gordon dont le matériau de base est constitué d'un long métrage que l'artiste se réapproprie pour questionner certains ressorts du dispositif cinématographique, tels que la vitesse de projection (vingt-quatre images par seconde) et l'ellipse temporelle dans 24 Hour Psycho (1993), basée sur Psychose (1960) d'Alfred Hitchcock. Concernant l'œuvre de Douglas Gordon, voir la monographie de Katrina M. Brown, Douglas Gordon, Londres, Tate Publishing, 2004. Plus récemment, le MoMA de New York a consacré une exposition monographique à Tim Burton, preuve supplémentaire des échanges de plus en plus fréquents entre musée et cinéma: "Tim Burton", 22 novembre 2009 - 26 avril 2010. Voir le site internet de l'exposition: www.moma. org/interactives/exhibitions/2009/timburton/.

12 Sous le concept d'"entre-image", Bellour étudie le passage entre image fixe et image mobile, les rapports entre cinéma, vidéo et autres domaines artistiques ainsi que les mutations de l'image que ces échanges impliquent: “[...] la vidéo retrouvant de l'impressionnisme au cubisme et au-delà les questions de la peinture, nous entraîne ici sur la terra incognita d'une nouvelle détermination du visuel (pour ne s'en tenir qu'à l'image), entre cinéma et peinture, en vue d'un autre corps-mémoire." (Raymond Bellour, "Thierry Kuntzel et le retour de l'écriture", Cahiers du cinéma, n 321 , mars 1981, p. 49).

13 Pascale Cassagnau, Future Amnesia Enquêtes sur un troisième cinéma, Paris, Editions Isthme, 2007, p. 11.

14 Pour un état critique de la question des rapports entre cinéma et art contemporain, voir l'article de François Bovier, "Des stratégies artistiques du réemploi à l'outil de production Anna Sanders Films", Décadrages. Cinéma, à travers champs, $\mathrm{n}^{\circ} 13$, automne 2008, pp. 9-16. 
15 Par exemple dans Ever Is Over All (1997), installation audiovisuelle, 2 projecteurs, 1 système audio, vidéos de $4^{\prime}$ et $9^{\prime}$ en boucle. Les deux projections se chevauchent sur deux murs perpendiculaires d'une pièce plongée dans l'obscurité. Le fait que la projection recouvre l'angle de convergence des deux murs lui confère du relief. La vidéo de la projection de gauche présente un personnage féminin se promenant dans les rues d'une ville. Cette femme fait tout d'abord penser à une héroïne de dessin animé ou de conte pour enfants avec sa robe bleue claire, ses chaussures à talons d'un rouge vif et l'énorme fleur qu'elle tient dans les mains. Cette première impression est mise à mal lorsque la jeune femme emploie la fleur comme une arme pour briser les vitres d'une voiture. Ce noyau narratif est développé lorsqu'une agente de police croise la femme et ne réprimande pas son acte mais la salue. La vidéo de la projection de droite est constituée de gros plans d'éléments végétaux.

\section{Pickelporno, 1992, 12'.}

17 J'emprunte le concept d'"attraction " à Tom Gunning qui l'a convoqué pour traiter du cinéma des premiers temps, d'avant 1907, qui fait primer le visuel sur le narratif: "Les attractions s'opposent sur plus d'un point à la construction narrative. D'abord, elles s'adressent directement au spectateur, et éveillent son attention ou sa curiosité par l'acte de monstration. En tant que moment de spectacle, elles focalisent l'attention et ne cherchent pas à développer les données de base de la narration [...]." (Tom Gunning, "Cinéma des attractions et modernité ", Cinémathèque, $\mathrm{n}^{\circ} 5$, printemps 1994 , p. 130). Ce mode visuel peut aussi se retrouver dans des films d'autres périodes, ce pourquoi je me permets d'y recourir ici.

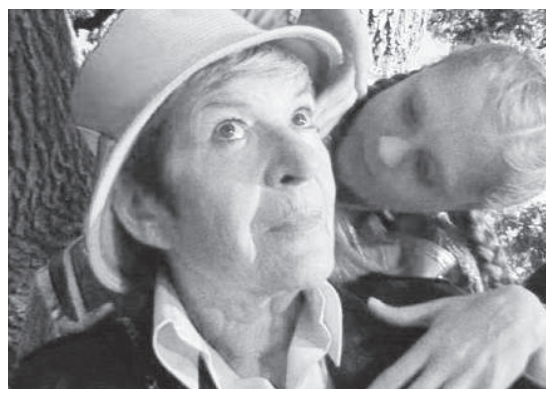

2/ Rencontre entre Pepperminta et Leopoldine qui va mener à une scène d'"onirisme vidéo". Source: The Match Factory d'installation est déjà présent un lien au cinéma par le recours à la projection en grand format ou la création de petites cellules narratives $\mathbf{1 5}$. Avec Pepperminta, l'artiste va plus loin. Il n'est plus seulement question de mobiliser une technique du cinéma ou un ressort du film mais de recourir directement à la forme du long métrage et au dispositif cinématographique. L'appropriation n'est toutefois pas totale, l'entre-deux persiste. Les couleurs saturées, la mobilité extrême de la caméra, certains motifs ou thématiques rappellent différentes vidéos et installations de l'artiste. Bien plus qu'un simple rappel, il s'agit d'un véritable travail d'autocitation.

\section{La logique de l'autocitation}

L'hypothèse selon laquelle Pepperminta repose sur un processus d'autocitation implique la présence d'un "avant-film", actualisé selon différentes modalités. Un travail précis de recherche puis d'analyse est nécessaire pour établir si le long métrage n'est qu'une suite de reprises d'éléments tirés de la vidéo ou s'il constitue l'approfondissement et l'adaptation d'une démarche artistique initiée en vidéo. Ce que nous proposons de regrouper sous l'appellation d'«avant-film» rassemble des séquences précises d'œuvres vidéo, des thématiques et le personnage de Pepperminta.

L'impression générale qui ressort du premier visionnement de Pepperminta est celle d'un fort lien esthétique avec l'œuvre de Pipilotti Rist. Une seconde approche plus analytique permet de mettre en évidence l'existence de séquences créées à partir d'un réemploi direct de vidéos précises. C'est le cas de la scène de la rencontre entre Pepperminta et Leopoldine, la vieille dame qui craint la mort (fig. 2). Leopoldine assise sur un banc, dans une forêt, est approchée par Pepperminta qui commence par caresser le visage puis les vêtements de la femme. Un changement de plans fait abruptement passer à une suite de gros plans sur les corps nus des deux femmes. Les doigts de Pepperminta passent sur les mains et la poitrine de Leopoldine, images qui sont très proches d'une vidéo réalisée par l'artiste en 1992, Pickelporno ${ }^{16}$. On y trouve le même filmage à fleur de peau de deux corps qui se rencontrent. L'unique différence réside dans la manipulation de la représentation convenue, hétérosexuelle du désir. L'opposition à la norme par la présentation d'un couple homosexuel est approfondie par la nudité d'une vielle femme, le dévoilement d'un corps âgé s'opposant au corps jeune et beau qui est habituellement représenté dans ce type d'images. Le recours à une vieillarde réduit le potentiel érotique de la rencontre. Comme Pickelporno, la séquence de Pepperminta est de l'ordre de la pure attraction $\mathbf{1 7}$. Rien ne justifie narrativement que les deux femmes soient d'abord 
vêtues et en discussion puis que soudain elles se retrouvent allongées dans l'herbe totalement nues. L'attirance physique aurait très bien pu être intégrée à l'histoire de Pepperminta. Dans le cinéma "conventionnel» mais aussi pornographique, que convoque dans cette scène Pipilotti Rist, le désir mène à une multitude de récits. Cette façon d'insérer des séquences d'attraction à l'intérieur du flux narratif est récurrente dans tout le long métrage. Nous retrouvons d'ailleurs une seconde actualisation de Pickelporno. Le fond végétal aux couleurs modifiées et saturées sur lequel flottent les corps nus des personnages de Pepperminta dans l'une de ses séquences oniriques $\mathbf{1 8}$ est quasiment identique à celui de la vidéo.

La dimension onirique de l'histoire de Pepperminta rend possible l'insert de telles séquences d'attraction. Les nombreuses séances de relaxation ou de communion psychique que l'héroïne et sa troupe effectuent avant d'entrer en action ajoutent une seconde strate au film. Au pan narratif est associé un pan mental où la part de fantaisie et d'abstraction est augmentée. Cet univers fantasmé est dépeint par des moyens très proches de ceux que Pipilotti Rist emploie dans ses vidéos. Le traitement de l'image y est volontairement révélé par des couleurs très vives, saturées et par des incrustations de motifs.

L'autoréférence ne s'arrête pas au niveau visuel, elle touche également des composantes narratives telles que certaines thématiques et les personnages. Le motif du sang menstruel est traité à plusieurs reprises dans le film et a suscité de vives réactions dans la presse. Ce thème a d'abord fait l'objet d'une vidéo en 1993, Blutclip ${ }^{\mathbf{1 9}}$. La première scène où apparaît le sang menstruel de Pepperminta réemploie plusieurs aspects de cette œuvre. On y retrouve un corps féminin nu sur lequel s'écoule du sang menstruel et un filmage très proche du corps qui met en évidence le contraste entre la blancheur de la peau et le rouge du liquide corporel. La principale adaptation réside dans un ralentissement du mouvement de la caméra qui sert un but narratif. La concentration de la caméra sur une coulée de sang permet de montrer que Pepperminta le recueille dans un calice. Les images participent pleinement à la narration. La menstruation constitue l'un des principaux moteurs du récit20. La volonté initiée dans Blutclip de lui conférer une valeur positive est approfondie dans Pepperminta. Ses potentialités de traitement visuel sont poursuivies et l'idée d'une puissance lui étant intrinsèque est totalement actualisée. Le sang menstruel devient la base de deux rituels et la clé de la victoire de la troupe de Pepperminta sur les peurs des gens. La narrativité nécessaire au long métrage permet un développement de la thématique menstruelle qui n'était pas possible dans le cadre de la vidéo.
18 Cette séquence onirique intervient après que Pepperminta et sa troupe ont fui le restaurant gastronomique qu'ils ont mis sens dessus-dessous. La benne qu'ils utilisent comme moyen de transport termine sa course dans l'eau. Les personnages flottent dans un univers aquatique et se mettent à ôter leurs vêtements. Je qualifie d'"oniriques" les séquences qui ne sont pas liées à la narration ou qui le sont de manière très vague.

19 Blutclip, 1993, 2' 30".

20 Est révélateur de son importance dans l'économie narrative du film sa mention dans le dossier de presse du film. Pipilotti Rist lie sang menstruel et puissance de création. Voir dossier de presse, op. cit., p. 8. 
21 Homo Sapiens Sapiens, 2005, installation audiovisuelle, 4 projecteurs placés dans des enceintes en direction du plafond, 4 lecteurs, 1 synchroniseur, 1 système sonore, coussins, vidéo de $21^{\prime}$ en boucle. Pipilotti Rist a représenté la Suisse avec cette installation lors de la Biennale de Venise de 2005. Dans cette première version, quatre projections recouvrent, sans se chevaucher et sans espace entre elles, la surface exacte du plafond voûté de l'église San Stae de Venise. Le visiteur est invité à ôter ses chaussures et à se coucher sur des coussins répartis sur le sol de l'église. L'architecture du lieu confère à l'installation une dimension presque mystique qui va de pair avec le thème du Jardin d'Eden traité dans la vidéo.

22 A Liberty Statue for Löndön, 2005/2008, installation audiovisuelle, version monolithe avec 3 projecteurs, 3 lecteurs, 1 système sonore, 1 surface miroir sur le sol entourée de couchettes et d'oreillers en velours, vidéo de 9' 37" en boucle. II s'agit de la version exposée dans la section "Art Unlimited" d'Art Basel 2008. L'installation se présente sous la forme d'un cube dans lequel le visiteur entre. A l'intérieur, il est totalement isolé du reste de l'espace d'exposition et découvre une projection au plafond et un sol recouvert de velours rouge sur lequel il est invité à s'allonger. Les projecteurs sont dissimulés à l'intérieur du rectangle en miroir disposé sur le sol et autour duquel les spectateurs se couchent. L'image du plafond est composée de l'association de trois projections. Elle est formée par deux couches, une image ronde au centre, accompagnée par une seconde de forme cubique en arrière-plan. La projection se reflète dans le miroir, ce qui permet au spectateur de la regarder au plafond ou au sol. Informations aimablement fournies par Karin Seinsoth de la galerie Hauser \& Wirth Zurich.

23 Expression tirée de la présentation de l'œuvre sur le site internet de l'exposition "Elixir: The Video Organism of Pipilotti Rist" qui s'est tenue au Museum Boijmans Van Beuningen de Rotterdam du 7 mars au 10 mai 2009. Voir le site internet du musée: www.boijmans. $\mathrm{nl} / \mathrm{en} / 161 /$ exhibition/exhibition/124/elixir-thevideo-organism-of-pipilotti-rist/5/.

24 En 2007, Pepperminta réapparaît dans Gravity Be My Friend, installation audiovisuelle, 2 projecteurs, 3 lecteurs, 1 système sonore, 2 sculptures en tapis, 2 écrans, vidéos de 11' et 12 ' en boucle. Lors de l'exposition "Elixir: The Video Organism of Pipilotti Rist" au Museum

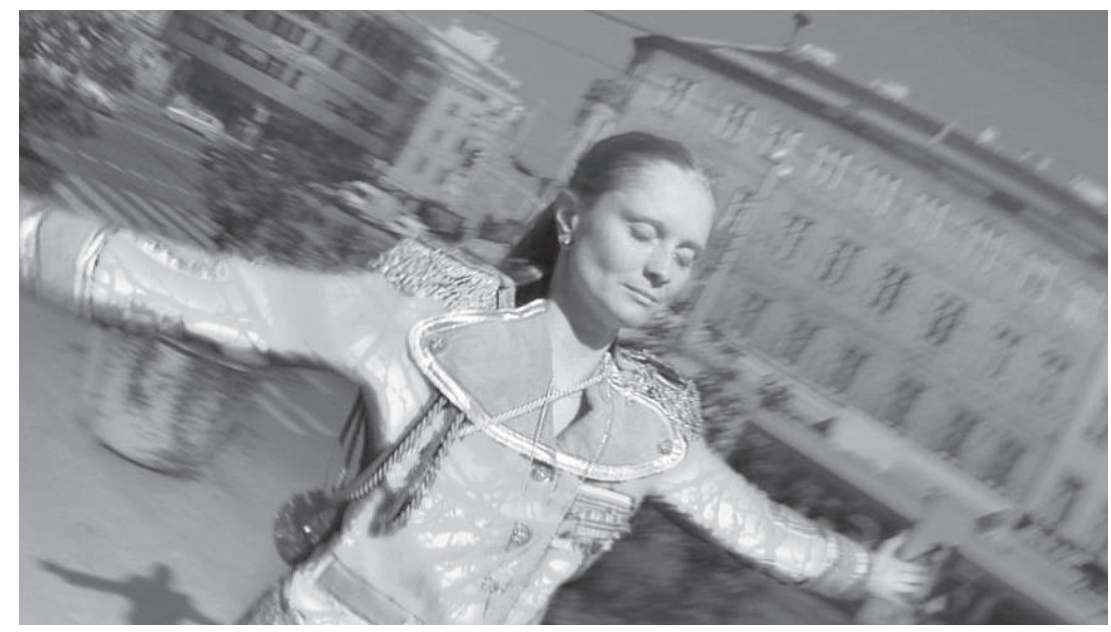

3/ Pepperminta plongée dans son univers mental. Source: Frenetic Films

Le personnage de Pepperminta fait également l'objet d'une actualisation qui dépasse le simple réemploi. L'actrice Ewelina Guzik qui prête ses traits à Pepperminta n'en est pas à sa première apparition dans l'univers de Pipilotti Rist. Les deux femmes ont débuté leur collaboration en 2005 avec l'installation Homo Sapiens Sapiens $\mathbf{2 1}$ puis A Liberty Statue for Löndön 22. Elle l'ont poursuivie jusqu'à aujourd'hui, notamment en 2007 avec les installations Gravity Be My Friend et $A$ la belle étoile et en 2009 par Pour Your Body Out (7354 Cubic Meters). C'est toujours Ewelina Guzik qui incarne le personnage principal que l'artiste appelle déjà «Pepperminta». Dans la vidéo de Homo Sapiens Sapiens, Pepperminta est une jeune femme qui évolue accompagnée de sa sœur dans un univers proche du paradis. Le corps nu de la femme rousse flotte sur un fond végétal. Une suite de gros plans se concentre sur son visage, ses mains et ses pieds. Pepperminta est une sorte d'Eve d'avant la Chute, d'être humain originel qui vit dans un environnement dépourvu de références temporelles et de classes sociales. Elle est en totale harmonie avec le monde qui l'entoure, communie avec la nature en étalant la pulpe de fruits bien mûrs sur sa peau. Pepperminta apparaît à nouveau dans l'installation A Liberty Statue for Löndön. Il ne s'agit plus de la «Pepperminta préhistorique» 23 de Homo Sapiens Sapiens. La jeune femme passe d'une forêt automnale magique à la civilisation, comme en témoignent le décor urbain dans lequel elle se trouve et les vêtements qu'elle porte. Bien qu'elle ne soit plus nue, la caméra se concentre à nouveau sur des parties visibles de son corps: son visage, ses mains et sa chevelure ${ }^{24}$. Quatre ans plus tard, Pepperminta évolue dans un long métrage de fiction cinéma- 
tographique. Le fait que le film porte le nom de son héroïne témoigne du développement dont a profité le personnage. Il ne s'agit plus d'une icône féminine de l'origine, ni d'un corps de femme employé pour ses seules potentialités esthétiques $\mathbf{2 5}$. Pepperminta est devenue un personnage de fiction à part entière (fig. 3). Elle est bien établie dans la civilisation contemporaine qu'elle a pénétrée dans A Liberty Statue for Löndön. Son autoprésentation au début du film donne quelques informations, bien que passablement abstraites, sur sa personnalité. Pepperminta a une histoire, rapportée à travers plusieurs flashbacks qui permettent de comprendre comment elle est devenue cette jeune femme anticonformiste. En un mot, le film dote ce personnage d'une profondeur psychologique qu'il ne possédait pas dans son traitement en vidéo. Comme le motif du sang menstruel, le personnage de Pepperminta sort du registre de l'attraction, du poème visuel propre à la vidéo, pour entrer dans celui de la narration cinématographique $\mathbf{2 6}$.

\section{L'autocitation poursuivie: l'" après-film " sous forme d'installation(s)}

Pour Pipilotti Rist, la salle de cinéma est «une forme différente d'espace d'installation" qui présente toutefois de "plus grandes difficultés» $\mathbf{2 7}$. Elle actualise ce lien entre médiums dans plusieurs œuvres réalisées parallèlement au tournage de Pepperminta 28. Quelques jours avant la

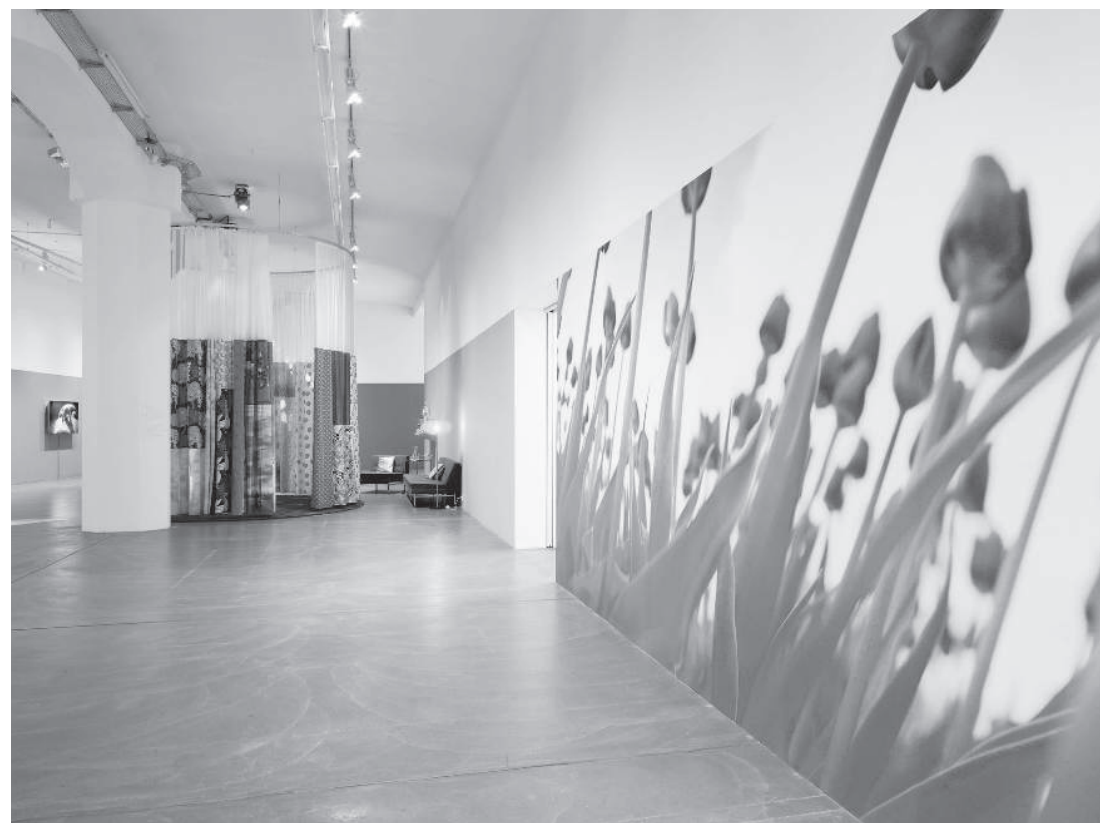

Boijmans Van Beuningen de Rotterdam, l'installation prenait la forme suivante: Deux vidéos sont projetées sur deux surfaces informes, aux contours très sinueux, fixées au plafond. Au-dessous de celles-ci sont disposées deux sculptures en tapis à la forme et aux dimensions semblables aux deux écrans. Le visiteur peut s'y allonger pour se relaxer en regardant les projections au plafond. Les vidéos présentent Pepperminta accompagnée d'un second personnage masculin, les deux corps représentent l'androgynie d'un être humain originel et volent, flottent en apesanteur au-dessus du monde. Au sujet du personnage de Pepperminta, voir Richard Julin (éd.), Pipilotti Rist. Congratulations!, Stockholm/Baden, Magasin 3 Stockholm Konsthall/Lars Müller Publishers, 2007, p. 57.

25 II importe toutefois de remarquer que Pepperminta est présentée selon une esthétique presque identique dans les vidéos et dans le long métrage. Cette constante visuelle s'explique en grande partie par la collaboration de Pipilotti Rist avec le même caméraman, Pierre Mennel.

26 Pipilotti Rist établit à plusieurs reprises un parallèle entre vidéo et poésie, et cinéma et narration ou roman, notamment dans le dossier de presse du film: "Si, avant de réaliser Pepperminta, j'écrivais au moyen de mon œuvre de la poésie, alors ce film est ma première histoire. " (Je traduis à partir du dossier de presse, op. cit., p. 8).

\section{$27 \mathrm{lbid}$.}

28 John Slyce parle d'une "famille issue du matériau filmique réalisé durant le tournage de Pepperminta". (John Slyce, "Pipilotti Rist. Adventures Close to Home ", dans John Slyce et al., Elixir: The Video Organism of Pipilotti Rist, Rotterdam, Museum Boijmans Van Beuningen, 2009, p. 51).

4/ Décors floral et installation Wohnzimmer ohne Angst accueillant le visiteur de l'exposition "Pipilotti Rist " à la galerie Hauser \& Wirth de Zurich. Pipilotti Rist, vue des installations à la galerie Hauser \& Wirth Zurich (2009). Source: Stefan Altenburger Photography Zurich 
5/ Pipilotti Rist, vue des installations à la galerie Hauser \& Wirth Zurich (2009). Source: Stefan Altenburger Photography Zurich
29 "Pipilotti Rist", 29 août - 17 octobre 2010, galerie Hauser \& Wirth, Zurich. Voir le site internet de la galerie qui propose une visite virtuelle de l'exposition: www.hauserwirth.com/ exhibitions/323/pipilotti-rist/video/.

La concordance entre la période de l'exposition et la sortie du film dans les cinémas alémaniques fait penser à une sorte d'" avant-première muséale".

30 "L'imagerie dans l'installation provient d'images prises par l'artiste durant le tournage de son premier long métrage Pepperminta (2009)." (Je traduis à partir du dossier de presse de l'exposition "Pipilotti Rist", disponible à l'adresse internet suivante: cloud.hauserwirth.com/documents/pipilotti-rist_pressrelease3-HCNOmV.pdf).

31 "Installation pour un salon ", projection lumineuse, tapis, rideau, dimensions variables. Cette installation était placée au centre de l'espace d'exposition, face à l'entrée de la galerie pour accueillir le visiteur. Un rideau suspendu au plafond formait un espace cylindrique dont le sol était recouvert de tapis et illuminé par un projecteur. A côté de l'installation était disposé un lecteur de disques audio.

32 Ursula Badrutt Schoch, "Pipilotti Rist - Wir sind hier, um uns die Schmerzen zu erleichtern ", Kunstbulletin, n 10, octobre 2009, p. 35.

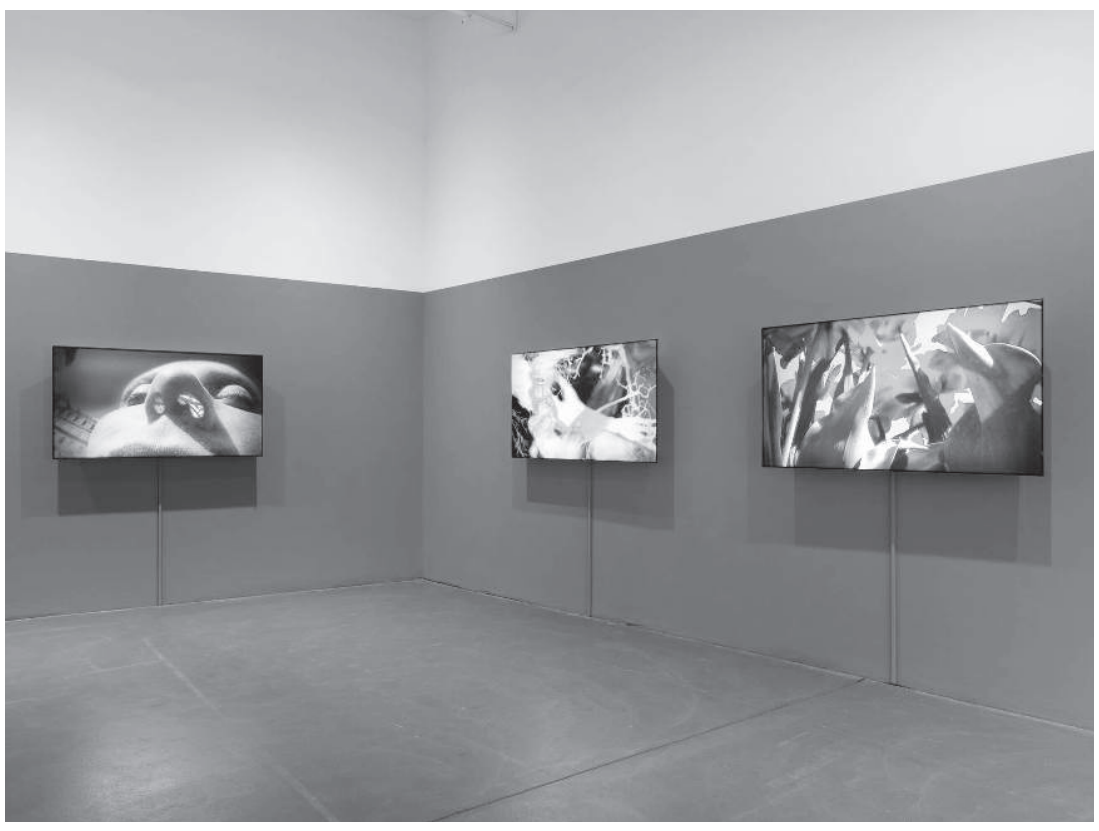

sortie du film en Suisse allemande, la galerie Hauser \& Wirth a verni à Zurich une exposition monographique consacrée à l'artiste29. Malgré l'absence du nom Pepperminta dans les titres des œuvres, le dossier de presse met clairement en évidence l'origine filmique de l'exposition $\mathbf{3 0}$. Des thèmes et des motifs visuels du film sont isolés et deviennent des œuvres autonomes.

La thématique centrale du film, la peur, fait l'objet de l'installation interactive Wohnzimmer obne Angst (2009) $\mathbf{3 1}$ (fig. 4). "Mélange de cabine d'essayage et de scène de danse» $\mathbf{3 2}$, cette œuvre isole le spectateur du reste de l'espace d'exposition et l'invite à se débarrasser de ses angoisses et tensions en dansant au rythme de la musique de son choix. Les couleurs de Pepperminta sont présentées non plus sous forme liquide mais sous forme lumineuse. Un projecteur mobile diffuse un faisceau lumineux multicolore qui croise le regard du visiteur et achève la libération initiée par le son. La luminosité se retrouve dans une série d'installations murales composées de photogrammes appliqués sur des caissons lumineux. Ce groupe d'œuvres est complété par des impressions couleur sur bois et sur papier. Le lien à Pepperminta n'y est plus d'ordre thématique mais visuel. On retrouve le visage, les mains et d'autres parties du corps de l'héroïne ainsi que le décor végétal du film. Le point commun entre ces images réside dans les dimensions des éléments représentés. Le cadrage 
est identique à celui du film ou encore plus resserré. La proximité à l'objet rapproche ces gros plans de l'abstraction. Si l'on se concentre sur le rapport au long métrage, on remarque que les images sélectionnées par l'artiste sont tirées de séquences oniriques, lorsque le visuel supplante le narratif. Les couleurs saturées et le traitement de l'image suggèrent plus une esthétique vidéo que cinématographique. Les œuvres de cette exposition approfondissent la part d'attraction présente dans Pepperminta, le pan vidéo ou art contemporain de l'entre-deux qu'il véhicule (fig. 5).

Le potentiel d'installation de Pepperminta développé dans l'exposition zurichoise a été approfondi par Pipilotti Rist dans une installation autonome: Lungenflügel (2009)33. L'image en mouvement de cette œuvre établit un lien plus direct avec la forme du long métrage cinématographique. La réutilisation et la manipulation touchent à la fois la forme et le fond. Des scènes précises sont reprises, retravaillées et mélangées à d'autres ne figurant pas dans Pepperminta mais provenant des chutes du film. Lorsque la citation touche des scènes narratives, c'est le potentiel visuel des images qui est développé. Dans la reprise de la séquence de la guérison de Werwen par Pepperminta, Pipilotti Rist se concentre sur les gros plans de tulipes qui sont très proches de l'abstraction. Quand il s'agit de moments oniriques, leurs liens à la narration sont supprimés. Ces scènes sont isolées et présentées comme autonomes. Les images liées à la seconde scène de relaxation de la troupe de Pepperminta par exemple, où l'on voit l'héroïne nue se déplacer dans l'herbe à la manière d'un cochon, sont présentées dans une suite continue; le montage alterné les liant au fil narratif du film est éliminé. Pipilotti Rist a inséré parmi ces extraits de Pepperminta des éléments dont le lien au film est plus ardu à établir. Des gros plans de parties corporelles sont si retravaillés qu'ils sont plus proches de l'abstraction de certaines vidéos de l'artiste que de son long métrage. Pour faire de Pepperminta une installation audiovisuelle, Pipilotti Rist en a isolé le contenu attractionnel, a développé ses ressources visuelles en mettant de côté le rapport à la narration. Cette manipulation a un impact sur l'expérience proposée au public. Le spectateur de Lungenflügel n'est plus privé de sa mobilité. L'artiste contente ainsi pleinement les amateurs d'art, laissant les critiques de cinéma et les cinéphiles sur leur faim.

La boucle de l'autocitation est bouclée. Le film de cinéma cite des œuvres vidéo et propose au spectateur un rapport qui se rapproche de celui que l'on établit face à une installation. L'art contemporain est amené au cinéma puis le cinéma est intégré dans le domaine de l'art par l'exposition en galerie et l'installation Lungenflügel. Le régime de l'entre-deux persiste dans le passage d'un médium à un autre et dans
33 Installation audiovisuelle, 3 projecteurs, 3 lecteurs synchronisés, 1 système sonore, 30 oreillers, tapis. La triple projection occupe l'entièreté de la surface de trois murs d'une pièce rectangulaire. L'espace est laissé vide pour la circulation des visiteurs qui peuvent s'allonger sur des coussins dispersés sur le sol. L'œuvre a été exposée à Rotterdam, au Museum Boijmans Van Beuningen, et à Helsinki, au Museum of Contemporary Art Kiasma, lors de l'exposition "Elixir. The video organism of Pipilotti Rist ". Une troisième version de l'installation a été présentée en janvier 2010 lors du Sundance Film Festival de Park City. Informations aimablement fournies par Tamara Voser de l'Atelier Rist Sisters.

Pipilotti Rist avait déjà utilisé le matériau visuel accumulé durant le tournage de Pepperminta pour créer en 2008 l'installation Pour Your Body Out (7354 Cubic Meters) commandée par le MoMA. Installation audiovisuelle multicanale, enceintes pour projecteurs, éléments formant un siège circulaire, tapis. Une projection multiple recouvre les murs de l'atrium du MoMA et crée une sorte de paysage dans lequel peut s'immerger le visiteur. Au centre de l'espace se trouve une île constituée de fauteuils sur lesquels le visiteur peut s'asseoir ou s'allonger avant de reprendre sa flânerie dans l'espace de l'installation. Le visiteur a la possibilité d'expérimenter l'œuvre depuis l'intérieur mais peut aussi avoir une vision d'ensemble de l'installation depuis les étages supérieurs du musée. Voir le site internet du musée: www.moma.org/ visit/calendar/exhibitions/307. 
le croisement des domaines de création. La spécificité du premier long métrage de Pipilotti Rist réside dans l'importance de son contenu intertextuel et de sa dimension intermédiale. Une telle complexité nécessite une flexibilité dans l'approche du film pour que soit possible le voyage à travers les champs du cinéma et de l'art contemporain auquel nous convie Pepperminta.

Pepperminta (Suisse/Autriche, 2009, 84'). Réalisation: Pipilotti Rist. Scénario: Chris Niemeyer, Pipilotti Rist. Image: Pierre Mennel. Montage: Gion-Reto Killias. Musique : Anders Guggisberg, Roland Widmer. Interprétation: Ewelina Guzik (Pepperminta), Sven Pippig (Werwen), Sabine Timoteo (Edna), Elisabeth Orth (Leopoldine), Noëmi Leonhardt (Pepperminta enfant), Oliver Akwe (Kwame). Production: Hugofilm Productions Zurich, Coop99 Vienne. Distribution en Suisse: Frenetic Films. 